\title{
SCHEDULING REQUESTS ON MULTI-STAGE MULTI-SERVER TO INCREASE QUALITY OF SERVICE
}

\author{
Ali Allahverdi \& Fawaz S. Al-Anzi \\ College of Engineering and Petroleum, Kuwait University, P.O. Box 5969, Safat, Kuwait, \\ Email:allahverdi@kuc01.kuniv.edu.kw,alanzif@eng.kuniv.edu.kw
}

Abstract: In this paper, we consider the problem of scheduling on a multi-stage multiserver architecture in computer centers to enhance quality of service through minimizing the average completion time of a set of requests. This problem is known to be an NP-hard combinatorial problem. We show that the problem can modeled as a flexible flowshop problem for which few heuristics exist in the flowshop scheduling literature. We propose a new heuristic for this problem that consists of three phases. Simulated Annealing is used in the first phase of the heuristic followed by a greedy algorithm phase. The last phase is a local pair-wise exchange procedure. An extensive computational experiment has been conducted to compare the existing heuristics with each other for the first time. Moreover, the proposed heuristic is compared with the existing heuristics. The results indicate that the proposed heuristic significantly outperforms the existing ones.

Key words: Scheduling, Multi-Stage, Multi-Server, Quality of service

\section{INTRODUCTION}

One of the important factors in the determining the quality of an internet service is the average response time of the requests. Typically an internet service requires the processing of a request coming from different clients on a multi stage architecture where each stage usually consists of a pool of parallel processors (servers) performing the same operation. The number of parallel servers in a stage is determined by how critical this particular stage

The original version of this chapter was revised: The copyright line was incorrect. This has been corrected. The Erratum to this chapter is available at DOI: 10.1007/978-0-387-35703-4_21

D. Gaïti et al. (eds.), Network Control and Engineering for QoS, Security and Mobility II

(C) IFIP International Federation for Information Processing 2003 
is to the overall quality of the service. This architecture is widely used in the internet since it provides flexibility in scalability and robustness.

When a service request arrives to such an architecture it follows a predetermined order of different stages of the service. Usually the amount of processing time of the request can be known before hand. At any point of time, there are usually a set of service requests waiting to be processed. The order of providing the service to the available set of requests significantly affects the average response time of the service. Therefore, it is essential to determine the best order for processing this set of requests in order to minimize the average response time. This performance measure ensures fairness to different clients.

Although this is an on-line problem where requests are arriving in a period of time, we can use a static version of the problem where there is a fixed number of requests. This model is valid since the requests are collected until the system becomes available from the previous batch of requests. Once it becomes available the batch of accumulated requests are considered for processing next. Hence, this can be considered as a static system within a window of time that is equivalent in duration to the time taken to process the previously collected batch of requests.

This problem is precisely the same as the flexible multi-stage flowshop problem with the objective of minimizing the average completion time. This problem has been addressed in the literature in the context of machine scheduling, and it is known to be NP-hard [1]. Therefore, implicit enumeration techniques, such as the branch-and-bound, have been proposed for a small number of jobs, see [2] and [3]. For larger number of jobs, efficient heuristics are required. The literature survey reveals that Sridhar and Rajendran [4], Brah and Loo [5], and Azizoglu et al. [6] presented different heuristics. The problem has also been addressed in the context of machine scheduling for other performance measures such as makespan which denotes the completion time of the last request. This performance measure is useful in the context of multi-server internet services for situations where the set of requests are parts of a single transaction where the transaction cannot be considered complete unless all the requests are successfully executed. Recent work with makespan performance measure includes Cheng et al. [7], Choi and Lee [8], Moursli and Pochet [9], Grabowski and Pempera [10], Gendreau et al. [11], and Negenman [12].

In this paper, we propose a new heuristic for the problem with the average completion time performance measure. The proposed heuristic consists of three phases. Simulated Annealing is used in the first phase of the heuristic followed by a greedy algorithm phase. The last phase is a local pairwise exchange procedure. An extensive computational experiment has been conducted to compare the existing heuristics of Sridhar and Rajendran [4], Brah and Loo [5], and Azizoglu et al. [6] with each other for the first 
time. Moreover, our proposed heuristic is compared with the existing heuristics. The results indicate that the heuristic by Sridhar and Rajendran [4] is practically the best among the existing ones. The results also indicate that our proposed heuristic significantly outperforms the existing ones in terms of the average error, the number of best obtained solutions, and the standard deviation of the error.

\section{PROBLEM DEFINITION}

Consider the configuration where there are $m$ stages. Stage $j(j=1,2, \ldots, m)$ consists of pj identical parallel servers, see Figure 1. There is a set of $n$ requests available for scheduling. Every request $i$ is to be processed by one of the servers in stage 1 then one of the servers in stage 2 and so on until it is processed by one of the servers in stage $\mathrm{m}$. If all the servers at a stage are busy, then a request has to wait until one of the servers at that stage becomes available. A server at a given stage can only process one request at a time and no preemption is allowed.

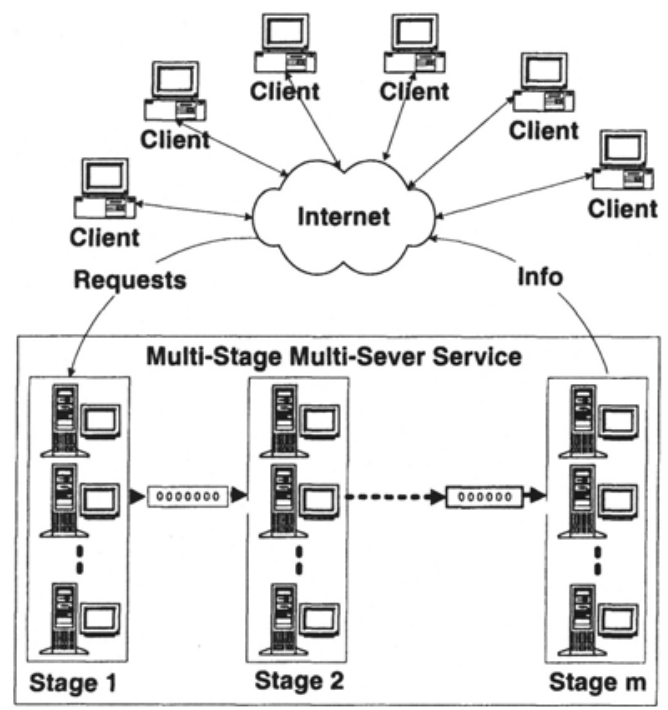

Figure 1. A typical multi-stage parallel-processor Internet service architecture

The model can be algebraically defined as follows:

Let

$\mathrm{n}$ : the number of requests,

$\mathrm{m}$ : the number of stages,

$p_{j}$ : the number of parallel servers at the $\mathrm{j}^{\text {th }}$ stage, 
$\mathrm{tk}_{\mathrm{j}}$ : the processing time of the request at the $\mathrm{k}^{\text {th }}$ position on the $\mathrm{j}^{\text {th }}$ stage,

$\mathrm{A}_{\mathrm{ij}}$ : the earliest time that the $\mathrm{i}^{\text {th }}$ server at the $\mathrm{j}^{\text {th }}$ stage is available,

$\mathrm{C}_{\mathrm{ij}}$ : the completion time of the request at position $\mathrm{i}$ at the $\mathrm{j}^{\text {th }}$ stage.

$A_{t}$ the beginning, all the servers are available since no request is being processed. Therefore,

$$
A_{i j}=0 \text { for all } i \text { and } j \text {. }
$$

We assume that a request at position $\mathrm{i}$ is processed by the earliest available server at the $\mathrm{j}^{\text {th }}$ stage, e.g., the $\mathrm{r}^{\text {th }}$ server at the $\mathrm{j}^{\text {th }}$ stage. Then, the completion time of a request at position $i$ can be expressed as

$$
C_{i 1}=A_{r 1}+t_{i j}
$$

where $A_{r 1} \leq A_{k 1}$ for $k=1,2, \ldots, p_{1}$ and $k \neq r$ for $j=1$. For $j=2,3, \ldots, m$,

$\mathrm{C}_{\mathrm{ij}}=\max \left\{\mathrm{C}_{\mathrm{ij}}-1, \mathrm{~A}_{\mathrm{rj}}\right\}+\mathrm{t}_{\mathrm{ij}}$

for $\mathrm{k}=1,2, \ldots, \mathrm{p}_{\mathrm{j}}$, and $\mathrm{k} \neq \mathrm{r}$ where $\mathrm{A}_{\mathrm{rj}} \leq \mathrm{A}_{\mathrm{kj}}$.

The availability is updated each time $\mathrm{C}_{\mathrm{ij}}$ is computed according to the following

$$
A_{r j}=C_{i j} \text { for } j=1,2, \ldots, m
$$

for the rth server that processed the request at position $\mathrm{i}$.

The total and average completion times (TCT, ACT) of all requests can be expressed as

$$
\begin{gathered}
T C T=\sum_{i=1}^{n} C_{i, m} \\
A C T=(1 / n) \sum_{i=1}^{n} C_{i, m}
\end{gathered}
$$

The objective is to find a schedule of requests that minimizes ACT. Notice that the TCT objective function is equivalent to ACT objective function since $1 / n$ is a constant for a given set of $n$ requests.

\section{EXISTING HEURISTICS}

The flexible multi-stage flowshop problem is the generalization of the multi-stage flowshop problem where there is only one server at each stage. It is known that even the two-stage flowshop problem with the average completion time performance measure is NP-hard, see Gonzalez and Sahni [1]. Therefore, the problem addressed in this paper is also NP-hard demonstrating the need for efficient heuristics. The literature review reveals that Sridhar and Rajendran [4], Brah and Loo [5], and Azizoglu et al. [6] are the only ones establishing heuristics for our problem. In the following, the existing heuristics are described. 


\subsection{ACK Heuristic}

Azizoglu et al. [6] presented a branch-and-bound algorithm to find an optimal solution. They proposed three heuristics to find an upper bound for their branch-and-bound algorithm. The three heuristics are as follows:

Heuristic 1. Stages are considered separately. At each stage, the problem is solved by using the Shortest Processing Time rule. The sequences found are imposed over all stages and the sequence that gives the minimum average completion time is selected.

Heuristic 2. The same as Heuristic 1 except that the sequence is only used at the first stage. For the other stages, among the available requests assign the request with the shortest processing time to the earliest available server.

Heuristic 3. The requests are ordered in a sequence according to a sequence proposed by Rajendran and Chaudhuri [13] of a non-decreasing order of $\sum_{j=1}^{m}(m-j+1)_{t j} j$ where $\mathrm{i}$ denotes a request. Requests are assigned to the earliest available server at each stage.

In this paper, all the three heuristics are evaluated and the best of the three is chosen. This is denoted as ACK heuristic.

\subsection{HO Heuristic}

Brah and Loo [5] have applied the existing heuristics designed for the regular flowshop problems to flexible flowshop problems with different performance measures including average completion time. They found that the heuristic by Ho [14] performed the best among the others with respect to average completion time minimization. The $\mathrm{HO}$ heuristic is as follows:

1. Let $\mathrm{k}=1$

2. Obtain an initial sequence by arranging the requests in ascending order of $\sum_{j=1}^{m}(m-j+1) t_{i j}$ for every request $\mathrm{i}$

3. Sort the initial solution using the bubble sort and call it the current solution.

4. Set $Z_{1}=A C T$

5. a. Sort the current solution by the insertion sort

b. Sort the current solution by the non-adjacent pair-wise interchange method

6. Sort the current solution by the bubble sort

7. Set $Z_{2}=A C T$

8. If $k<n-4$, and $Z_{1} \neq Z_{2}$ then $k=k+1$, and go to Step 4 , otherwise the current solution is the final solution.

The insertion and bubble sort methods are well known sorting algorithms in computer science, see Knuth [15]. 


\subsection{SR Heuristic}

Sridhar and Rajendran [4] were the first to propose a heuristic for the problem. They proposed a three phase heuristic. An initial seed sequence is obtained in the first phase by using the Rajendran and Chaudhuri [13] algorithm. This seed sequence is used as a starting sequence for the Simulated Annealing algorithm. Their heuristic is referred to as SR Heuristic in this paper.

\section{PROPOSED HEURISTIC (PH)}

Our proposed heuristic $\mathrm{PH}$ is described in this section. It consists of three phases. In phase one, an initial sequence is obtained by applying a simulated annealing algorithm. In phase two, the sequence obtained from phase one is improved by a repeated application of the greedy algorithm. Finally, in phase three, the sequence acquired from phase two is further improved by a local pairwise exchange procedure. The description of each phase of $\mathrm{PH}$ heuristic is explained next.

\section{Phase I: Simulated Annealing Algorithm}

1.Get a random initial sequence $\pi_{1}$

2. Set an initial temperature $\mathrm{T}$

3.If $\mathrm{T}<\mathrm{TF}$ go to step 9

4.Repeat steps 5 to $8 \mathrm{k}$ times

5.Perform a random pairwise exchange to $\pi_{1}$, and call the resulting sequence as $\pi_{2}$

6.Let $\mathrm{D}=\mathrm{ACT}\left(\pi_{2}\right)-\mathrm{ACT}\left(\pi_{1}\right)$

7. If $D \leq 0$, then set $\pi_{1}=\pi_{2}$, otherwise

7.1. $\mathrm{p}=\mathrm{e}^{-\mathrm{D} / \mathrm{T}}$

7.2. Set $\pi_{1}=\pi_{2}$ with the probability $p$

8. Set $\mathrm{T}=\mathrm{T}^{*} \mathrm{r}$ where $\mathrm{r}$ is temperature updating factor, go to step 3 .

9. Select the best solution $\pi_{1}$ and set it as the current sequence.

\section{Phase II: Greedy Algorithm}

Here we describe the two parts of the greedy algorithm of phase II separately.

Greedy Algorithm I

1. Given an input sequence of $\mathrm{n}$ requests.

2. Set $\mathrm{k}=1$ and current solution to be empty 
3. Generate $\mathrm{k}$ candidate sequences by splitting the current solution into two parts at all possible $\mathrm{k}$ positions and inserting the $\mathrm{k}^{\text {th }}$ request in the merged sequence.

4. Compute partial ACT for all $\mathrm{k}$ candidate sequences. Among these candidates, select the one with the least ACT.

5. Update the one with the least ACT as a current solution.

6. Update $\mathrm{k}=\mathrm{k}+1$

7. If $\mathrm{k}=\mathrm{n}+1$ then stop, else go to Step 3 .

The Algorithm described above was first used by Nawaz et al. [16] for a different problem (regular flowshop) and a different performance measure (makespan).

\section{Greedy Algorithm II}

This algorithm makes $\log 2(\mathrm{n})$ passes on the list of requests. Here, we assume that $n$ is multiple of 2's. If not, we increase $n$ to the closest multiple of 2 and fill the rest of request slots by zero processing time requests which will be removed from the final request sequence. In pass number i, the list is evenly divided into subsequences, each of size $2^{\mathrm{i}-1}$. For every two subsequences $j$ and $j+1$ that are to be merged, we iterate the insertion of every element in the subsequence $j+1$ into the subsequence $j$ maintaining the best sequence that has the least average completion time in the produced subsequences.

1. Given an input sequence of $\mathrm{n}$ requests.

2. Set the input sequence as current sequence

3. For $\mathrm{i}=1, \ldots, \log _{2}(\mathrm{n})$ Repeat Steps 4-10.

4. Divide the current sequence into subsequences of size $2^{\mathrm{i}-1}$.

5. For every two subsequences $j$ and $j+1$ that will be merged repeat Steps 6-9.

6. For every request $\mathrm{k}$ in subsequence $\mathrm{j}+1$ from left to right.

7. Generate candidate sequences by infusing the $\mathrm{k}$ request into each possible slot of $\mathrm{j}$ subsequence.

8. Compute the partial ACT for each candidate sequence. Among these candidates, select the one with the least ACT.

9. Update the one with the least ACT as the $\mathrm{j}$ sequence.

10. Join all subsequences resulting for Steps 7-9 into one sequence and let it be the current sequence.

\section{Phase III: Local Pairwise Exchange}

1.Set $\mathrm{i}=1$

2.Compute $\mathrm{ACT}$ of the current sequence, and call it $\mathrm{ACT}_{1}$

3. Exchange the requests in position $i$ and $i+1$

4. Compute ACT of the current sequence, and call it $\mathrm{ACT}_{2}$

5.If $\mathrm{ACT}_{2}<\mathrm{ACT}_{1}$, then go to Step 7 
6. Exchange the requests in position $i$ and $i+1$

7. Set $\mathrm{i}=\mathrm{i}+1$

8.If $i<n$, then go to Step 2

Notice that the policy of assigning a sequence of the requests at each stage can be one of two policies. The first policy is to enforce the initial sequence (the sequence fed to stage one) to all the subsequent stages. The second policy is that a request departing from a previous stage is assigned to the earliest available server from the current stage. If there is more than one request available when a server is free, the priority is given to the one with the shortest processing time. In our proposed heuristic we adopt the second policy.

Setting the parameters for Phase I of our proposed heuristic is essential in achieving a good performance. After extensive experimentation, the parameters are set as follows; $\mathrm{T}=0.1, \mathrm{r}=0.98, \mathrm{TF}=0.0001$, and $\mathrm{k}=50$.

In Phase II, the greedy algorithms are applied in the sequence of Algorithm I-Algorithm II-Algorithm I repeatedly. This process is repeated for five times since no significant improvement was observed beyond this. It should be noted that there is no guarantee that each time a better solution will be obtained. Therefore, among the five repetitions the best one is always kept as the current solution. Experimentation has shown that the proper sequence of greedy algorithms is Algorithm I- Algorithm II - Algorithm I since other permutations did not perform as well. Experimentation has also shown that using Algorithm II or Algorithm I alone did not perform well.

Similarly Phase III is repeated and it has been observed that five repetition of the local pairwise exchange procedure was sufficient to produce a good solution.

It can be shown that the time complexity of phases I, II, and III are $\mathrm{O}(\mathrm{nm}), \mathrm{O}\left(\mathrm{n}^{2} \mathrm{~m} \log \mathrm{n}\right)$, and $\mathrm{O}\left(\mathrm{n}^{2} \mathrm{~m}\right)$, respectively. Therefore, the time complexity of $\mathrm{PH}$ is $\mathrm{O}\left(\mathrm{n}^{2} \mathrm{~m} \log n\right)$.

\section{HEURISTIC COMPARISON}

The three existing heuristics of ACK, HO, and SR along with our proposed heuristic $\mathrm{PH}$ were implemented in C on a Sun Sparc 20, and evaluated with respect to average error, standard deviation of the error, and the number of times yielding the best solution. We also evaluated the SR heuristic without its initial seed generation phase. This will leave their algorithm as a regular simulated annealing algorithm which is referred to as SA in this paper where the initial sequence is chosen randomly.

The processing times were randomly generated from a uniform distribution $(1,100)$. In the scheduling literature, most researchers have used 
this distribution in their experimentation, e.g., Al-Anzi and Allahverdi [17], and Allahverdi and Al-Anzi [18]. The reason for using a uniform distribution is that the variance of this distribution is large and if a heuristic performs well with such a distribution, it will certainly perform well with any other distribution.

Problem data are generated for different number of requests ranging from 20 to 100 in increment of 10 . The number of stages considered is 2 to 6 while at each stage the number of parallel servers is randomly generated from a uniform discrete distribution from 1 to 5 . We compare the performance of the heuristics for thirty replicates using three measures: average percentage error (Error), standard deviation (Std), and the number of times the best solution is obtained (PB). The percentage error is defined as 100* (Heuristic Error- Best Heuristic Error)/Best Heuristic Error. The Best Heuristic Error is the error of the heuristic yielding the minimum error.

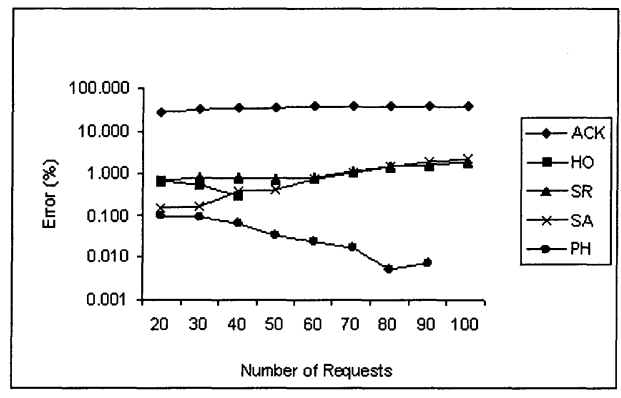

Figure 2. Error versus n

Figures 2 and 3 shows the results of running the existing and the proposed heuristics for all combinations of the number of requests and the number of stages. Each heuristic is evaluated for the same configuration to ensure accurate assessment of the different heuristics. The results for $\mathrm{HO}$ are not reported for the number of requests greater than 40 because of its large CPU time requirement with an inferior performance compared to PH. For example, for 40 requests and 6 stages, the CPU times (Sec) of ACK, HO, $\mathrm{SR}$, and $\mathrm{PH}$ were $0.029,278,32$, and 49 while the errors were $32.52,0.36$, 1.33 , and 0.1 , respectively. The CPU time of $\mathrm{HO}$ increases significantly as the number of requests increases while the CPU times of the other heuristics increase moderately. It should be noted that the HO heuristic only performs well for the case of two stages on the expense of heavy computational time.

Figures 2 illustrates the performance of the heuristics with respect to the number of requests. Due to the poor performance of ACK, Figures 2 and 3 have semi-logarithmic scale for more vivid comparison. It is clear that 
among the existing heuristics in the literature SR and HO significantly outperform ACK. Even though HO outperforms SR (at least for the number of requests less than 40), the heavy CPU time requirement of $\mathrm{HO}$ makes it impractical to use it for larger problems. Hence, SR can be considered practically the best among the existing ones. As can be seen from Figure 2, the average error of the proposed heuristic $\mathrm{PH}$ gets better as the number of requests increases while that of SR deteriorates. Due to the limited space the results for the other performance measures of the number of best solutions and standard deviation are not reported. However, it has been found that the same can be said for the other performance measures for the PH and SR heuristics. Therefore, the proposed $\mathrm{PH}$ heuristic is superior to the SR heuristic.

Figure 3 illustrates the performance of the heuristics with respect to the number of stages. Again, the semi-logarithmic scale is used in Figure 3 due to the poor performance of ACK. In general, for all number of stages, the proposed $\mathrm{PH}$ heuristic outperforms all the existing heuristics in terms of all the three performance measures. Again the results for the other performance measures of the number of best solutions and standard deviation are not reported due to limited space. It can be seen that the performance measures slightly deteriorate as the number of stages increases for all the heuristics.

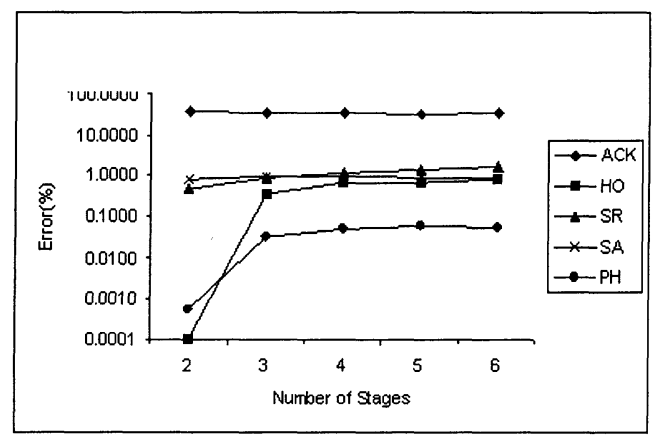

Figure 3. Error versus m

The comparison of heuristics SR and SA reveals that, in general, SA performs better for larger number of stages while SR performs better for small number of stages. Notice that the difference between SR and SA is that SR uses a computed initial seed while SA uses a random seed. Hence, the use of the calculated seed of SR is recommended for small number of stages. The reason for the good performance of SR for small number of stages might be that the priority used in finding the position of a request in the 
initial sequence is directly proportional to its processing time and inversely proportional to the stage number.

The overall average of Errors of ACK, HO, SR, SA and the proposed heuristic $\mathrm{PH}$ are $34.4,0.48,1.09,0.89$, and 0.04 , respectively. Clearly ACK is not recommended because of its extremely poor performance. $\mathrm{PH}$ outperforms HO since HO's error is 12 times the error of $\mathrm{PH}$ and the CPU time of $\mathrm{HO}$ is significantly higher than that of PH. PH also outperforms SR since the error of SR is 27 times that of $\mathrm{PH}$ while the CPU time of $\mathrm{PH}$ is only about 1.5 times that of SR. It should be noted that the performance of all the five heuristics with respect to the number of best solution and the standard deviation is similar to that of the average error. The overall average of percentage of best solutions (PB) of ACK, HO, SR, SA, and proposed heuristic $\mathrm{PH}$ are $0,40.44,14.44,10.59$, and 82.73 while the overall average Std are $10.3,0.63,0.87,0.36$, and 0.08 , respectively. Therefore, $\mathrm{PH}$ is superior to all the existing heuristics.

\section{CONCLUDING REMARKS}

The problem of scheduling on a multi-stage parallel-server architecture in computer centers is addressed in this paper with respect to the average completion time of a set requests. This problem can be modeled as a flexible flowshop problem. The flexible flowshop literature review reveals that there exist few heuristics to minimize average completion time. In this paper, we compare the existing heuristics with each other. Moreover, we propose a new heuristic for this problem that consists of three phases; Simulated Annealing Algorithm, greedy algorithm, and a local pairwise-exchange procedure. An extensive computational experiment has been conducted to compare the existing and proposed heuristics. The results indicate that the proposed heuristic significantly outperforms the existing ones.

Simulated Annealing is a well known optimization technique and hence it has many applications. This paper shows that the performance of simulated annealing technique can be enhanced significantly by applying greedy and local optimization techniques to its results. Moreover, since simulated annealing is a global optimization technique, selection of a computed seed needs not to produce a better result compared with a randomly selected seed.

The approach used in this paper can be extended to problems with other objective functions such as makespan and maximum lateness. 


\section{Acknowledgements}

This research was supported by Kuwait University Research Administration project number EE 01/01.

\section{REFERENCES:}

1. Gonzalez T, Sahni S. Flow shop and job shop schedules. Operations Research 1978; 26: 36-52.

2. Linn R, Zhang W. Hybrid flowshop scheduling: A survey. Computers and Industrial Engineering 1999; 37: 57-61.

3. Rajendran C, Chaudhuri D. A multi-stage parallel-processor flowshop problem with minimum flowtime. European Journal of Operational Research 1992; 57: 111-122.

4. Sridhar J, Rajendran C. Scheduling in a cellular manufacturing system: a simulated annealing approach. International Journal of Production Research 1993; 31: 2927-2945.

5. Brah SA., Loo LL. Heuristics for scheduling in a flow shop with multiple processors. European Journal of Operational Research 1999: 113: 113-122.

6. Azizoglu M, Cakmak E, Kondakci S. A flexible flowshop problem with total flow time minimization. European Journal of Operational Research 2001; 132: 528-538.

7. Cheng TCE, Lin BMT, Toker A.. Makespan minimization in the two-machine flowshop batch scheduling problem. Navel Research Logistics 2000; 47: 128-144.

8. Choi AH, Lee JSL. A sequence algorithm for minimizing makespan in multi-part and multi-machine flowshop case. Integrated Manufacturing Systems 2000; 11: 62-73.

9. Moursli O, Pochet Y. A branch-and-bound algorithm for the hybrid flowshop. International Journal of Production Economics 2000; 64: 113-125.

10. Grabowski J, Pempera J. Sequencing of jobs in some production system. European Journal of Operational Research 2000; 125: 535-550.

11. Gendreau M, Laporte G, Guimaraes EM. A divide and merge heuristic for the multiprocessor scheduling problem with sequence dependent setup times. European Journal of Operational Research 2001; 133: 183-189.

12. Negenman EG. Local search algorithms for the multiprocessor flow shop scheduling problem. European Journal of Operational Research 2001; 128: 147-158.

13. Rajendran C, Chaudhuri D. An efficient heuristic approach to the scheduling of jobs in a flowshop. European Journal of Operational Research 1991; 61: 318-325.

14. Ho JC. Flowshop sequencing with mean flowtime objective. European Journal of Operational Research 1995; 81: 571-578.

15. Knuth DE. The Art of Computer Programming: Sorting and searching, MA (USA): Addison-Wesley, 1973.

16. Nawaz M, Enscore E, Ham I. A heuristic for the m-machine, n-job flowshop sequencing problem. OMEGA 1983;11:91-95.

17. Al-Anzi F, Allahverdi A. The relation between three-tired client-server internet database and two-machine flowshop. International Journal of Parallel and Distributed Systems and Networks 2001; 4: 94-101.

18. Allahverdi A, Al-Anzi F. Using two-machine flowshop with maximum lateness objective to model multimedia data objects scheduling problem for WWW applications. Computers and Operations Research 2002; 29: 971-994. 